\title{
Papers
}

\section{Effects of grandmothers' smoking in pregnancy on birth weight: intergenerational cohort study}

\author{
Elina Hyppönen, George Davey Smith, Chris Power
}

\begin{abstract}
Objective To investigate the influences on birth weight of maternal smoking during pregnancy across generations.

Design Intergenerational cohort study.

Participants Members of the 1958 birth cohort and their offspring and mothers.

Setting England, Scotland, and Wales.

Main outcome measure Birth weight.

Results Information on grandmothers' smoking during pregnancy was available for 9028 singleton offspring of 4302 female cohort members. Assuming heritable transmission through the intergenerational association, grandmothers' smoking was predicted to result in a $34 \mathrm{~g}$ reduction (95\% confidence interval

$-41 \mathrm{~g}$ to $-28 \mathrm{~g}$ ) in the birth weight of grandchildren. Random effects models showed a negative association between grandmothers' smoking and birth weight of grandchildren ( $\beta$ regression coefficient $-24 \mathrm{~g},-50 \mathrm{~g}$ to $3 \mathrm{~g}$ ), but this effect was eliminated after adjustment for maternal smoking ( $0 \mathrm{~g},-26 \mathrm{~g}$ to $26 \mathrm{~g})$. No association was evident among the offspring of non-smoking mothers $(\mathrm{n}=6105 ; 14 \mathrm{~g},-17 \mathrm{~g}$ to $46 \mathrm{~g})$, and after adjustment for maternal birth weight, adult height and body mass index, grandmothers' smoking was positively associated with the birth weight of grandchildren ( $45 \mathrm{~g}, 10 \mathrm{~g}$ to $80 \mathrm{~g}$ ).

Conclusion Deficits in mothers' birth weight attributable to their mother smoking was not evident in the grandchildren.
\end{abstract}

\section{Introduction}

Evidence on the effects of exposure of fetuses to tobacco smoke has been restricted to two generations (parent and offspring), but there are reasons to expect that the effects of maternal smoking during pregnancy could extend to the third generation. The positive association between birth weights of mothers and their children are well known. ${ }^{12}$ The Dutch hunger winter study showed that the effects of famine during fetal life may be transmitted to subsequent generations through this intergenerational association. ${ }^{3}$ Birth weight associations between mothers and offspring have typically been found stronger than those between fathers and offspring. This has been taken to reflect maternal constraint on fetal growth: a restrictive mechanism determined in utero and transmitted through the female line to the next generations. ${ }^{5}$ Evidence to support regulation of fetal development by an intrauterine mechanism operating through the female progeny has been obtained from multigenerational studies in rhesus monkeys. ${ }^{6}$ The deleterious effect of maternal smoking on offspring stature and risk of obesity also suggests effects across generations. ${ }^{78}$ Maternal stature and obesity are recognised influences on birth weight, so it seems plausible that maternal smoking could have an indirect effect on the birth weight of offspring mediated through maternal size. ${ }^{19}$ We investigated whether maternal smoking during pregnancy reduces the birth weight of infants across more than one generation.

\section{Participants and methods}

We used data from the 1958 birth cohort. ${ }^{10}$ The cohort consisted of 16751 live births from 3 to 9 March 1958 in England, Scotland, and Wales. ${ }^{11}$ Detailed information on behavioural, social, and health indicators was collected at birth (98\% of cohort members) and at ages $7,11,16,23,33$, and $41 .{ }^{10}{ }^{12}$ Birth data were largely collected by midwives for cohort members (including birth weight in pounds and ounces and gestational age as days from the first day of the last menstrual period), and supplemented with information from obstetric records and interviews with the mothers. ${ }^{11}$ Information on smoking before and during the pregnancy was reported by the mothers of cohort members in 1958 and coded as smoking up to or after the fourth month of pregnancy. ${ }^{13}$ At age 33 and 41, cohort members provided data on their own offspring during an interview. They were asked if they had smoked during pregnancy. This was reported as not at all or up to or after the fifth month of pregnancy. Most (98\%) cohort members reported their offspring's birth weights in pounds and ounces. Information on the length of gestation of these offspring was obtained from inquiring if the child was born at term and, if not, by how many weeks he or she was early or late. Height of cohort members was measured at age 33, and supplemented with self reported values from surveys held at ages 23 and 41 . Height was reported to the nearest inch for all except 177 cohort members, who stated their height in centimetres at age 41 . We used the weight of cohort members as reported to the nearest pound at age $23 .{ }^{14}$
Centre for Paediatric Epidemiology and Biostatistics, Institute of Child Health, London WC1N 1EH Elina Hyppönen research fellow Chris Power professor of epidemiology and public health

Department of Social Medicine, University of Bristol, Bristol BS8 2PR

George Davey Smith professor of clinical epidemiology Correspondence to: E Hyppönen e.hypponen@ ich.ucl.ac.uk

bmj.com 2003;327:898 


\section{Participants}

We investigated the association between smoking in the mothers of cohort members and birth weight of their offspring among all singleton cohort members with birth data (88\% of singleton participants, 7023 females and 7466 males). Because data on smoking were not available for the partners of male cohort members, we restricted investigation of the effect of maternal smoking on birth weight in the next generation to singleton offspring of female cohort members. By age 41, 4615 of $6021(77 \%)$ female cohort members who were still being followed up had become mothers. Full information on birth weight, gestational age, and sex was obtained for 9372 liveborn singletons of 4471 female cohort members (97\%, range 1-9 children per woman). The mothers of 9028 offspring $(n=4302,93 \%)$ had full data on smoking (mothers and grandmothers) and birth characteristics (mothers and offspring). To eliminate the effect of smoking in cohort members on their offspring's birth weight, we repeated main analyses excluding the offspring of mothers who smoked at any time during pregnancy (2923 children of 1515 women, 35\%).

\section{Statistical analyses}

We used linear regression to estimate the deficit in birth weight of cohort members associated with smoking in their mother (regression coefficient $(\beta)$ for smoking after the fourth month of pregnancy compared with none, noted as $\mathrm{Y} 1 \mathrm{in}$ the formula below). We then assessed the association between birth weights of cohort members and their offspring by regressing the offspring's birth weight on that of the parent ( $\beta$ for each kilogram increase in offspring's birth weight by each kilogram in parent's birth weight, noted as Y2 below). The effects on offspring's birth weight was analysed with random effects models to allow for there being several offspring per mother. To obtain an expected level for the effects of maternal smoking across the generations, we calculated the predicted reduction in the offspring's birth weight according to smoking in the grandmother. In this calculation, the reduction in the cohort member's birth weight consequent on smoking in their mothers (Y1) was multiplied by the association between birth weight for cohort members and their offspring (Y2). Variance for this prediction was calculated as:

$\operatorname{Var}(\mathrm{Y} 1, \mathrm{Y} 2)=$

$\operatorname{Var}(\mathrm{Y} 1) * \mathrm{Y} 2^{2}+\operatorname{Var}(\mathrm{Y} 2) * \mathrm{Y} 1{ }^{2}+\mathrm{Y} 1 * \mathrm{Y} 2 * 2 * \operatorname{Cov}(\mathrm{Y} 1, \mathrm{Y} 2)$ in which $\operatorname{Cov}(\mathrm{Y} 1, \mathrm{Y} 2)=\operatorname{Cor}(\mathrm{Y} 1, \mathrm{Y} 2) * \mathrm{SE}(\mathrm{Y} 1) * \mathrm{SE}(\mathrm{Y} 2)$
We used $95 \%$ confidence intervals to compare the difference between the observed influence of grandmothers' smoking on grandchildren's birth weight (obtained from random effects models) and the predicted reduction in the cohort members' birth weight. Variance for the difference was the sum of observed and predicted variances.

Owing to the strong dependency of birth weight on length of gestation, we adjusted for gestational age in all models concerning cohort members' offspring. Analyses are for all children, adjusted for sex, because the effect of grandmothers' smoking on grandchildren's birth weight was similar for both sexes. To eliminate confounding by smoking in cohort members, the association between grandmother and grandchildren was adjusted for smoking in the cohort member. Analyses were repeated for the offspring of non-smoking cohort members. To determine the extent to which grandmothers' smoking influenced birth weight of grandchildren through size of the cohort member as an adult, we adjusted for height and body mass index (weight $(\mathrm{kg}) /\left(\right.$ height $\left.(\mathrm{m})^{2}\right)$ of the cohort member at age 23. As we had no information on maternal body mass index before pregnancy, a proxy measure was values obtained at age 23. Models including maternal body mass index were restricted to the majority (73\%) of births occurring after age 23 to avoid the influence of pregnancy on maternal body mass index. Further adjustments were made for cohort members' birth weight to account for genetic influences affecting the birth weight of their offspring.

\section{Results}

Overall, $35 \%$ of cohort members and $41 \%$ of their mothers smoked during pregnancy. Of these, smoking continued in $70 \%$ of cohort members after the fifth month of pregnancy and $82 \%$ of their mothers after the fourth month (table). In both generations, mothers who smoked during the final months of pregnancy had light offspring at birth. The average birth weight of cohort members' offspring differed little by grandmothers' smoking during pregnancy.

Each kilogram increase in maternal birth weight (cohort member generation) was associated with a $255 \mathrm{~g}$ increase $(95 \%$ confidence interval $226 \mathrm{~g}$ to $283 \mathrm{~g}$ ) in birth weight of their offspring, and given this intergenerational birth weight association grand-

Mean birth weight of offspring* by maternal and grandmaternal smoking during pregnancy

\begin{tabular}{|c|c|c|c|c|c|c|}
\hline \multirow[b]{2}{*}{$\begin{array}{l}\text { Smoking status during } \\
\text { pregnancy }\end{array}$} & \multirow[b]{2}{*}{ No } & \multirow{2}{*}{$\begin{array}{c}\text { Mean (SD) birth } \\
\text { weight of cohort } \\
\text { member }(g)\end{array}$} & \multicolumn{2}{|c|}{ Offspring of all women in cohort } & \multicolumn{2}{|c|}{ Offspring of non-smoking mothers in cohor } \\
\hline & & & No & $\begin{array}{c}\text { Mean (SD) } \\
\text { birth weight (g) }\end{array}$ & No & $\begin{array}{c}\text { Mean (SD) } \\
\text { birth weight (g) }\end{array}$ \\
\hline \multicolumn{7}{|l|}{$\begin{array}{l}\text { Mother of cohort } \\
\text { membert }\end{array}$} \\
\hline Non-smoker & 8597 & $3352(560)$ & 5240 & $3251(558)$ & 3761 & $3300(530)$ \\
\hline Before fourth month & 1074 & $3358(550)$ & 713 & $3244(541)$ & 486 & $3297(528)$ \\
\hline After fourth month & 4818 & $3181(581)$ & 3075 & $3211(551)$ & 1858 & $3298(528)$ \\
\hline Total & 14489 & $3295(572)$ & 9028 & $3237(554)$ & 6105 & $3299(529)$ \\
\hline \multicolumn{7}{|l|}{ Cohort member } \\
\hline Non-smoker & - & - & 6105 & $3299(529)$ & - & - \\
\hline Before fifth month & & & 720 & $3281(563)$ & & \\
\hline After fifth month & & & 2203 & $3048(577)$ & & \\
\hline Total & & & 9028 & $3237(554)$ & & \\
\hline
\end{tabular}

*All birth weights made to correspond to first born females by subtracting increase for males or greater parity.

tData on cohort member's birth weight by smoking in mother presented for all cohort members with birth data. Other numbers are based on participants with full data on smoking (cohort member and mother) and birth characteristics (cohort member and offspring). 
mothers' smoking was predicted to lead to a $34 \mathrm{~g}$ reduction $(-41 \mathrm{~g}$ to $-28 \mathrm{~g})$ in the birth weight of grandchildren. The random effects models suggested a lower birth weight in grandchildren if the grandmother smoked during pregnancy ( $\beta$ regression coefficient $-24 \mathrm{~g},-50 \mathrm{~g}$ to $3 \mathrm{~g}$ ), but this difference was eliminated after adjusting for smoking in the cohort member ( $0 \mathrm{~g},-26 \mathrm{~g}$ to $26 \mathrm{~g}$; fig 1). The observed value differed noticeably from the predicted reduction in birth weight of grandchildren by grandmothers' smoking after additional adjustment for the cohort member's birth weight, adult height, and body mass index at age 23 (difference $49 \mathrm{~g}, 19 \mathrm{~g}$ to $80 \mathrm{~g}$ ). When the analysis was restricted to the offspring of non-smoking female cohort members, grandmothers' smoking during pregnancy was not associated with the birth weight of offspring ( $\beta$ regression coefficient $14 \mathrm{~g}$, $-17 \mathrm{~g}$ to $46 \mathrm{~g}$ ), an observation that differed from that predicted (difference $52 \mathrm{~g}, 14 \mathrm{~g}$ to $90 \mathrm{~g}$; fig 2). Adjustment for maternal height or body mass index did not noticeably affect the association between grandmothers' smoking and birth weight in offspring. When the birth weight of the cohort member was adjusted for in addition to adult height and body mass index at age 23, there was a positive association between grandmothers' smoking and birth weight of grandchildren ( $\beta$ regression coefficient $27 \mathrm{~g},-3 \mathrm{~g}$ to $57 \mathrm{~g}$, and $45 \mathrm{~g}, 10 \mathrm{~g}$ to $80 \mathrm{~g}$, for offspring of all and non-smoking female cohort members, respectively), suggesting that the adverse influence of maternal smoking during pregnancy had been compensated for during transmission to the next generation. A weaker intergenerational birth weight association was suggested between cohort member and offspring if the grandmother had smoked during pregnancy, although the interaction between grandmothers' smoking and birth weight of the female cohort member was not significant ( $\beta$ for interaction $-12 \mathrm{~g}, \mathrm{P}=0.35$, and $-21 \mathrm{~g}$, $\mathrm{P}=0.19$, for all and non-smoking female cohort members, respectively). Among the offspring of nonsmoking cohort members, we found that if the grandmother had smoked during pregnancy, then each kilogram increase in the female cohort member's birth weight was associated with a $237 \mathrm{~g}$ (173 g to 300 g) increase in birth weight of their offspring, whereas a $277 \mathrm{~g}(233 \mathrm{~g}$ to $321 \mathrm{~g}$ ) increase was observed for the offspring of non-smoking grandmothers. Adjustment for height of grandmother or social class of the cohort member at birth and in adulthood did not noticeably affect the association between grandmothers' smoking and birth weight of grandchildren (data not presented).

\section{Discussion}

The association between maternal smoking and low birth weight of offspring does not seem to apply across more than one generation. We studied three generations centred on the 1958 birth cohort and found no association between grandmothers' smoking after the fourth month of pregnancy and birth weight of grandchildren. Our results suggest that the biological effects of this important environmental insult lowering birth weight are not transmitted across generations through the intergenerational birth weight association.

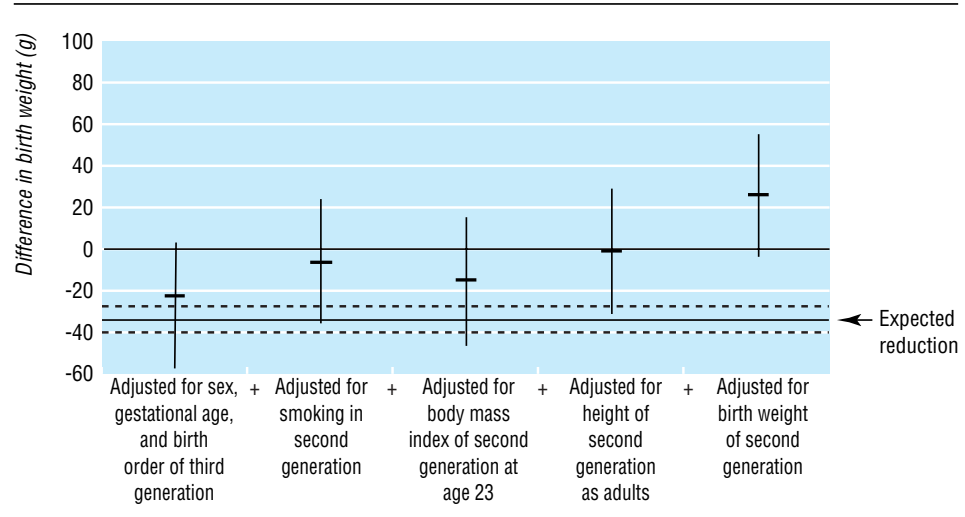

Fig 1 Birth weight of offspring of all mothers in 1958 birth cohort by grandmothers' smoking during pregnancy. 95\% confidence intervals are given by error bars for difference and by dashed lines for expected reduction

Earlier findings suggest that the short term effects of nutritional deprivation on the distribution of maternal birth weights may be carried over to a subsequent generation. ${ }^{3}$ In the Dutch hunger winter study, starvation during the first trimester of fetal life was associated with a slight increase in birth weight, whereas famine during the third trimester produced a substantial drop. These effects, although attenuated, were passed to the next generation through an intergenerational birth weight association. ${ }^{3}$ The Dutch study also suggested that adversity in early development has detrimental effects on subsequent health even if birth weight is not affected. ${ }^{15}$ In our study, we were able to examine only the effects of maternal smoking on the birth weight of offspring and grandchildren, and there may be a wide range of adverse health outcomes (possibly determined in early gestation) that do not depend on size at birth. It also seems possible that the effects on subsequent health of tobacco smoke may vary according to timing of exposure in utero. We were unable to distinguish between maternal smoking at different stages of pregnancy because the original data covered the period before and after the fourth month of pregnancy, and most women who smoked after the fourth month also smoked at the beginning of pregnancy.

Apart from the Dutch study, there is little information on how in utero influences affecting birth

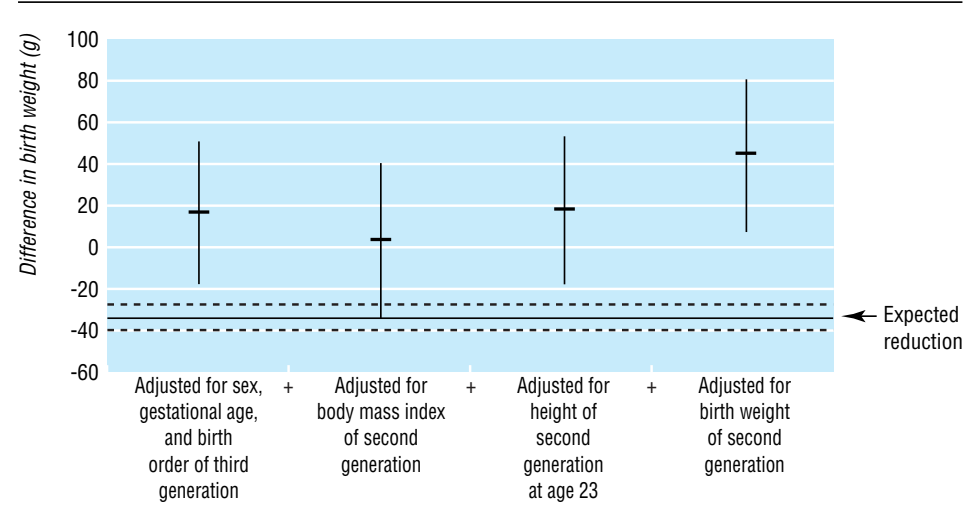

Fig 2 Birth weight of offspring of non-smoking mothers in 1958 birth cohort by grandmothers' smoking during pregnancy. $95 \%$ confidence intervals are given by error bars for difference and by dashed lines for expected reduction 
weight pass to subsequent generations. We assumed that the effect of grandmothers' smoking would be passed on to the extent predicted by the association between birth weights across generations. Although our calculation does not distinguish between transmissible and non-transmissible effects, it does show that environmental influences on birth weight weaken during transmission across generations. Furthermore, the positive association between grandmothers' smoking and birth weight of grandchildren after adjustment for maternal size suggests that there may be a compensatory mechanism for a deficit in birth weight as opposed to a transmissible effect.

Our study has some methodological limitations. In a large longitudinal study, such as the 1958 birth cohort that extends over several decades, losses to follow up are inevitable. The cohort has, however, been reported to be generally representative in most aspects until age 33. ${ }^{10}$ By including all participants with data on offspring in the surveys at age 33 or 41 , we were able to reduce the magnitude of non-participation. As our focus was on relations across generations, it seems unlikely that our results would have been affected by losses to follow up. ${ }^{4}$ Furthermore, we were limited to reported information on birth weights of grandchildren, even though the measures for cohort members were obtained from birth records. The recall period was, however, less than 15 years for $95 \%$ of participants, and it has been shown that maternal reports of birth weight are unbiased even after 20 to 40 years. $^{16}{ }^{17}$

\section{Conclusions}

We found that the reduction in birth weight of offspring attributable to maternal smoking was compensated for in the subsequent generation. Even if the effects had been transmitted, the predicted reduction in birth weight of offspring due to maternal exposure to tobacco smoke in the uterus was negligible.

We thank Jonathan Sterne and Tim Cole for statistical advice. Contributors: $\mathrm{EH}$ drafted the manuscript and carried out statistical analyses. She will act as guarantor for the paper. $\mathrm{CP}$ and GDS had the original study idea, and GDS developed the prediction calculation. All authors contributed to the final version of the paper.

Funding: The Wellcome Trust. The guarantor accepts full responsibility for the conduct of the study, had access to the data, and controlled the decision to publish.

Competing interests: None declared.

Ethical approval: Ethical committee approval for the 41 year survey was obtained from the North Thames MREC. Ethical approval was not sought for the survey at age 33, although cohort members were asked to give written consent for access to medical records.

\section{What is already known on this topic}

Little information exists on the long term influences of smoking on subsequent health of offspring

Maternal birth weight is positively associated with the birth weight of offspring

It is unclear whether the reduction in birth weight from exposure to tobacco smoke in utero is passed on through generations

\section{What this study adds}

A reduction in birth weight attributable to grandmothers' smoking was not passed on to grandchildren

Intergenerational birth weight associations depend on particular factors influencing birth weight

1 Emanuel I, Filakti H, Alberman E, Evans SJW. Intergenerational studies of human birth weight from the 1958 birth cohort. 1. Evidence for a multigenerational effect. Br J Obstet Gynaecol 1992;99:67-74.

2 Ramakrishnan U, Martorell R, Schroeder DG, Flores R. Role of intergenerational effects on linear growth.J Nutr 1999;129:544-9S.

3 Stein AD, Lumey LH. The relationship between maternal and offspring birth weights after maternal prenatal famine exposure: the Dutch famine birth cohort study. Hum Biol 2000;72:641-54

4 Hennessy E, Alberman E. Intergenerational influences affecting birth outcome. I. Birthweight for gestational age in the children of the 1958 British birth cohort. Paediatr Perinatal Epidemiol 1998;12:45-60.

5 Ounsted M, Scott A, Ounsted C. Transmission through the female line of a mechanism constraining human fetal growth. Ann Hum Biol 1986;13:143-51.

6 Price KC, Shibley Hyde J, Coe CL. Matrilineal transmission of birth weight in the Rhesus monkey (Macaca mulatta) across several generations. Obstet Gynecol 1999;94:128-34.

7 Fogelman K, Manor O. Smoking in pregnancy and development into early adulthood. BMJ 1988;297:1233-6.

8 Power C, Jefferis BJ. Fetal environment and subsequent obesity: a study of maternal smoking. Int J Epidemiol 2002;31:413-9.

9 Kramer MS. Determinants of low birth weight: methodological assessment and meta- analysis. Bull WHO 1987;65:663-737.

10 Ferri E. Life at 33: the fifth follow-up of the national child development study. London: National Children's Bureau, 1993.

11 Butler NR, Bonham DG. Perinatal mortality. Edinburgh: Livingstone, 1963.

12 National Child Development Study composite file including selected perinatal data and sweeps one to five [computer file]. Centre for Longitudinal Studies Institute of Education. National Birthday Trust Fund, National Children's Bureau, City University Social Statistics Research National Children's Bureau, City University Social Statistics Research
Unit [original data producers]. SN: 3148. Colchester, Essex: Data Archive distributor, 1994

13 Butler NR, Alberman E. Perinatal problems. Edinburgh: Livingstone, 1969 14 Lake JK, Power C, Cole TJ. Child to adult body mass index in the 1958 British birth cohort: associations with parental obesity. Arch Dis Child 1997;77:376-81.

15 Susser M, Stein Z. Timing in perinatal nutrition: a reprise of the Dutch famine study. Nutr Rev 1994;52:84-94.

16 Lumey LH, Stein AD, Ravelli AC. Maternal recall of birthweights of adult children: validation by hospital and well baby clinic records. Int J Epidemiol 1994:23:1006-12.

17 Sanderson M, Williams MA, White E, Daling JR, Holt VL, Malone KE, et al. Validity and reliability of subject and mother reporting of perinatal factors. Am J Epidemiol 1998;147:136-40.

(Accepted 31 July 2003) 